\title{
Integration of Tumor Mutation Burden and PD-L1 Testing in Routine Laboratory Diagnostics in Non-Small Cell Lung Cancer
}

\author{
Stefanie Schatz ${ }^{1,2,+}$ (D), Markus Falk ${ }^{1,2,+}$, Balázs Jóri ${ }^{3,+}$, Hayat O. Ramdani ${ }^{2,4,5}$, \\ Stefanie Schmidt ${ }^{1,2}$, Eva-Maria Willing ${ }^{3}$, Roopika Menon ${ }^{3}$, Harry J. M. Groen ${ }^{6}$, Linda Dieh1 ${ }^{5}$, \\ Matthias Kröger ${ }^{7}$, Claas Wesseler ${ }^{2,8}$, Frank Griesinger ${ }^{2,4,9}$, Petra Hoffknecht ${ }^{2,10}$, \\ Markus Tiemann ${ }^{1,2}$ and Lukas C. Heukamp ${ }^{1,2, *}$ \\ 1 Institut für Hämatopathologie Hamburg, Fangdieckstraße 75A, 22547 Hamburg, Germany; \\ schatz@hp-hamburg.de (S.S.); falk@hp-hamburg.de (M.F.); schmidt@hp-hamburg.de (S.S.); \\ mtiemann@hp-hamburg.de (M.T.) \\ 2 Lung Cancer Network NOWEL, 26129 Oldenburg, Germany; Hayat.Ramdani@Pius-Hospital.de (H.O.R.); \\ c.wesseler@asklepios.com (C.W.); Frank.Griesinger@Pius-Hospital.de (F.G.); \\ Petra.Hoffknecht@niels-stensen-kliniken.de (P.H.) \\ 3 NEO New Oncology GmbH, Gottfried-Hagen-Straße 20, 51105 Cologne, Germany; \\ Jori@newoncology.de (B.J.); Willing@hp-Hamburg.de (E.-M.W.); Menon@newoncology.de (R.M.) \\ 4 Department of Hematology and Oncology, Pius-Hospital Oldenburg, Georgstraße 12, \\ 26121 Oldenburg, Germany \\ 5 Institute of Experimental Immunology and Hepatology, University Medical Center Hamburg Eppendorf, \\ Martinistraße 52, 20246 Hamburg, Germany; li.diehl@uke.de \\ 6 Department of Pulmonary Diseases, University of Groningen and University Medical Center Groningen, \\ Hanzeplein 1, 9713 GZ Groningen, The Netherlands; h.j.m.groen@umcg.nl \\ 7 Onkologische Schwerpunktpraxis, Kröger Ambulante Onkologie, Wiener Straße 1, \\ 27568 Bremerhaven, Germany; praxiskroeger@googlemail.com \\ 8 Department of Internal Medicine and Pulmonology, Asklepios Klinikum Harburg, Eißendorfer \\ Pferdeweg 52, 21075 Hamburg, Germany \\ 9 Department of Internal Medicine-Oncology, University of Oldenburg, Georgstraße 12, \\ 26121 Oldenburg, Germany \\ 10 Germany Department of Thorax Oncology, Niels-Stensen-Kliniken, Franziskus-Hospital Harderberg Alte \\ Rothenfelder Straße 23, 49124 Georgsmarienhütte, Germany \\ * Correspondence: heukamp@hp-hamburg.de or heukamp@nowel.org; Tel.: +49-176-200-56495 \\ + Authors contributed equally.
}

Received: 30 April 2020; Accepted: 20 June 2020; Published: 24 June 2020

\begin{abstract}
In recent years, Non-small cell lung cancer (NSCLC) has evolved into a prime example for precision oncology with multiple FDA-approved "precision" drugs. For the majority of NSCLC lacking targetable genetic alterations, immune checkpoint inhibition (ICI) has become standard of care in first-line treatment or beyond. PD-L1 tumor expression represents the only approved predictive biomarker for PD-L1/PD-1 checkpoint inhibition by therapeutic antibodies. Since PD-L1-negative or low-expressing tumors may also respond to ICI, additional factors are likely to contribute in addition to PD-L1 expression. Tumor mutation burden (TMB) has emerged as a potential candidate; however, it is the most complex biomarker so far and might represent a challenge for routine diagnostics. We therefore established a hybrid capture (HC) next-generation sequencing (NGS) assay that covers all oncogenic driver alterations as well as TMB and validated TMB values by correlation with the assay (F1CDx) used for the CheckMate 227 study. Results of the first consecutive 417 patients analyzed in a routine clinical setting are presented. Data show that fast reliable comprehensive diagnostics including TMB and targetable alterations are obtained with a short turn-around time. Thus, even complex biomarkers can easily be implemented in routine practice to optimize treatment decisions for advanced NSCLC.
\end{abstract}


Keywords: immuno-oncology; tumor mutational burden; lung cancer; routine diagnostics; driver mutation; PD-L1

\section{Introduction}

In advanced non-small cell lung cancer, immune checkpoint inhibitors (ICIs) targeting the PD-1/PD-L1 axis, have sustainably changed the therapeutic approach of driver mutation negative tumors. ICIs have become available for first- and second-line treatment and represents a next step in the effort to reduce the group of patients receiving systemic chemotherapy. Despite these advances, a limited group of about $20 \%$ of NSCLC patients still benefit from ICI. Biomarkers are needed to predict the response outcome before initiation of therapy. As of now, PD-L1 expression has been established as the only predictive biomarker for treatment with ICIs; nevertheless, several factors are limiting its predictive value. Firstly, tumor heterogeneity in terms of PD-L1 tumor expression can be significant and can also change in response to therapy. Secondly, PD-L1-negative tumors have been reported to respond to ICI as well, rendering PD-L1 an imperfect biomarker and urging the need for other biomarkers, such as microsatellite instability (MSI) and TMB [1-7]. Potentially, TMB may be an independent factor from PD-L1 expression in predicting outcome. TMB is referred to as the total number of somatic mutations that occur in an exome of a tumor genome, although the exact calculation and definition of TMB might differ based on the type of variants, region size, or localization [8,9]. Exonic mutations can lead to the translation of novel peptide epitopes on the tumor surface that may increase the immunogenicity and, therefore, trigger an immune response [9-13]. TMB has been demonstrated to be a strong predictive value for the efficacy of ICI in second-and third-line monotherapy, in first-line monotherapy, and in the first-line combination of immuno-oncological (IO) substances, even if the patients' tumors did not show expression of PD-L1 [14-17]. Furthermore, TMB is evaluated for the selection of patients who benefit from the combination of nivolumab and ipilimumab over platinum-based chemotherapy [18]. A recent study described TMB as a predictor for survival after immunotherapy across multiple cancer types, including head and neck, bladder, breast, and renal cancer, underlining the general validity of TMB stratification [19]. Two other recent studies revealed that the efficacy of pembrolizumab plus chemotherapy or placebo plus chemotherapy as first-line treatment was not associated with TMB [20,21]. The lack of correlation between PD-L1-expression and TMB status has been observed in several different studies, highlighting the independent and potential complementary role for both biomarkers [14-16,22]. However, the measurement and calculation of TMB still misses uniform standards. Several factors can influence $\mathrm{TMB}$, including the DNA repair capacity and mutation rate. Because current neoantigen-predictive algorithms are imperfect, it is likely that the relationship between TMB and antigenicity is complex. Furthermore, additional factors can affect immunogenicity, including the clonality of neoantigens and the tumor microenvironment [9]. In addition, tumor heterogeneity and clonal architecture, the size of the selected genomic region of interest, and setup regarding driver mutational panel bias or even tumor purity can also influence the results from a biological perspective. From the technical point of view, NGS-derived deamination artifacts, the lack of predefined cut-off values; and the mutation types challenge the introduction of TMB as a general biomarker for immunotherapies [23]. The clinical utility of TMB is also affected by pre- and postanalytic parameters, such as storage of the formalin fixed paraffin embedded (FFPE) sample, turn-around time (TAT), or analysis failure rate [24,25]. To evaluate the importance of TMB as a practical biomarker, clinical routine data are needed. Here, we present the first TMB mono-centric dataset from a cohort of 417 lung cancer samples in Germany. We aimed to cover the proposed gaps by discussing clinical usability as driver mutations and TMB in the period from 2018 to 2020 using a commercially available assay (NEOplus v2 RUO ${ }^{\ddagger}$, NEO New Oncology $\mathrm{GmbH})$. The assay was designed to detect targetable driver mutations and genomic alterations (i.e., translocations, copy number changes) of other clinically relevant genes, such KRAS, KEAP1, STK11, 
and ARIDA1 (Appendix A), as well as to estimate TMB in an exonic territory of $1.14 \mathrm{Mb}$. After internal validation, the assay was used in our accredited clinical laboratory for routine mutation analysis in NSCLC patients. Samples were exclusively tested upon request by the attending physician and therefore reflect real world data

\section{Results}

\subsection{TMB Assay Correlation with Clinical Trail Assay of CheckMate 227}

To determine whether the assay was able to estimate TMB comparable to the assay used in the CheckMate 227 clinical trial, a series of 17 samples were analyzed both in-house as well as by F1CDx (Foundation Medicine Inc., Cambridge Massachusetts)

A high correlation $\left(\mathrm{R}^{2}=0.884,95 \% \mathrm{CI}[0.799,0.968]\right)$ between both assays was observed and the TMB category (high vs. low) showed 80\% (12/15) concordance with the F1CDx category with a cut-off of $10 \mathrm{mut} / \mathrm{Mb}$ (Figure 1).

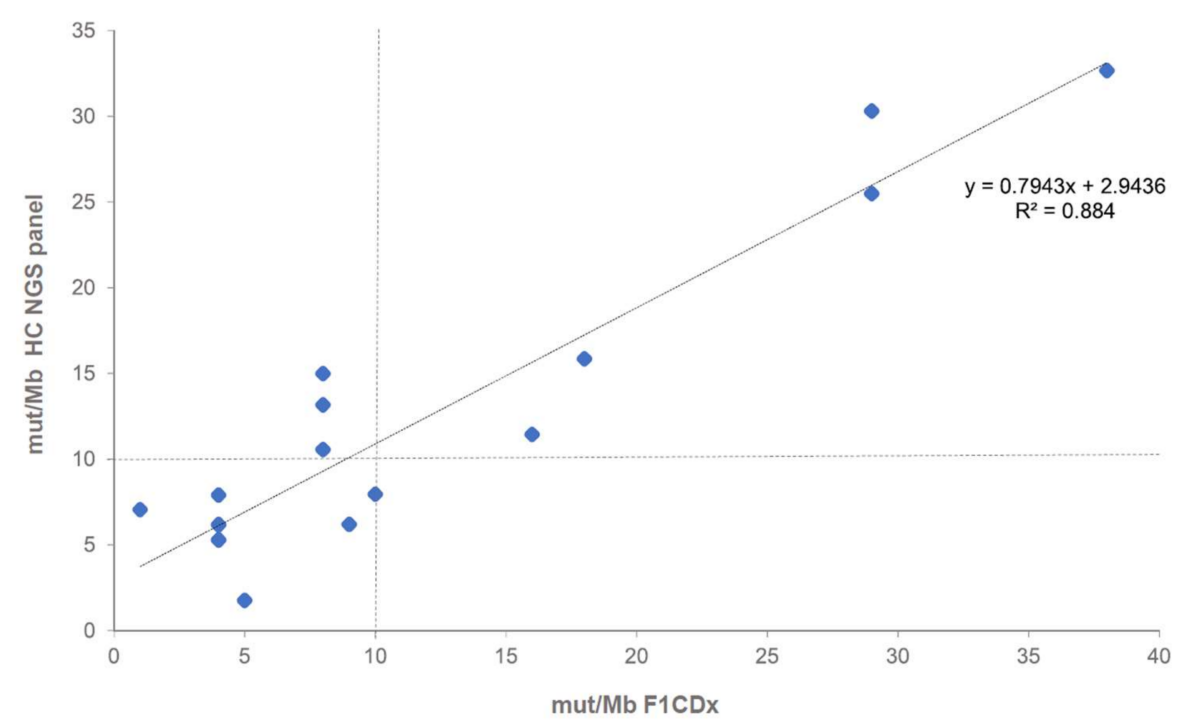

Figure 1. Correlation of TMB estimation of 17 samples measured by NEOplus v2 RUO $\ddagger$ and F1CDx assays.

\subsection{Histology, Driver Mutations, and PD-L1}

In this cohort, $42.4 \%$ (177/417) of patients were of female gender and $57.6 \%(240 / 417)$ were male, the mean age being 66 years (Table 1). Histological classification revealed 73.9\% (308/417) adeno carcinomas, $0.2 \%$ (1/417) adeno-squamous, $7.9 \%$ (33/417) squamous cell, 1.0\% (4/417) small cell lung cancer, $0.5 \%$ (2/417) of cases displayed large cell neuroendocrine differentiation, while $16.5 \%$ (69/417) were not otherwise specified (NOS). EGFR mutations were detected with a frequency of $14.87 \%$ (62/417); however, only 66\% (41/62) constituted the classical targetable drivers including exon 19 deletions or L858R. The remaining 34\% were mostly located outside exons 18-21. Exon 20 insertions, resistant to first-, second-, and third-generation EGFR tyrosine kinase inhibitors (TKIs), were detectable in six cases $(6 / 417,1.4 \%)$ (Table 1$)$.

Several point mutations were detected within the $B R A F$ gene $(36 / 417,8.63 \%)$; among these, nine were BRAF V600E. Deemed targetable gene fusions were found with a frequency of $9.83 \%(41 / 417)$, including 15 (3.66\%) EML4-ALK, 2 CD74-ROS1 (0.48\%), and 3 RET translocations (0.73\%, 2x KIF5B-RET, RET-CCDC6). Several other fusions were detected, including inter- and intragenic fusions of TP53, $R B 1, S T K 11$, and $C D K N 2 A / B$. Within the group of adenocarcinomas, $79.9 \%$ of cases were devoid of a targetable driver alteration and $1.6 \%$ showed wildtype in all 39 therapeutically relevant genes. None of the squamous cell carcinomas showed a targetable driver alteration. In parallel to mutational analysis, 
PD-L1 immunohistochemistry (IHC) was performed and the tumor proportion score (TPS) was determined for each sample. In total, 123/417 (29.5\%) of tumors did not express PD-L1 ( $<1 \%$ of PD-L1), while $11.99 \%$ showed PD-L1 expression between $\geq 1 \%$ and $<5 \%$ PD-L1, $18.94 \%$ between $\geq 5 \%$ and $<50 \%$ PD-L1, and $23.74 \%$ of cases had strong PD-L1 expression ( $\geq 50 \%$ PD-L1). In $15.8 \%$ of cases, no PD-L1 analysis was performed either because it was not requested by the physician or due to limited tissue availability.

Table 1. Overview of patients' characteristics according to TMB values.

\begin{tabular}{|c|c|c|c|c|c|c|c|}
\hline & \multirow{2}{*}{ Variation } & \multirow{2}{*}{\multicolumn{2}{|c|}{$\begin{array}{c}\text { Total } \\
N=417\end{array}$}} & \multicolumn{2}{|c|}{$\mathrm{TMB}<10$} & \multicolumn{2}{|c|}{$\mathrm{TMB}>10$} \\
\hline & & & & $N=260$ & $(62.35 \%)$ & $N=157$ & $(37.65 \%)$ \\
\hline & Median & \multicolumn{2}{|c|}{66} & \multicolumn{2}{|c|}{66} & \multicolumn{2}{|c|}{66} \\
\hline \multirow{4}{*}{ Age } & Mean $( \pm S D)$ & 65.0 & $(11.8)$ & 64.7 & $(12.5)$ & 65.7 & $(10.5)$ \\
\hline & Range & \multicolumn{2}{|c|}{$21-92$} & \multicolumn{2}{|c|}{$21-92$} & \multicolumn{2}{|c|}{$93-90$} \\
\hline & $<65$ years & 199 & $(47.72 \%)$ & 126 & $(48.46 \%)$ & 73 & $(46.50 \%)$ \\
\hline & $\geq 65$ years & 218 & $(52.28 \%)$ & 134 & $(51.54 \%)$ & 84 & $(53.50 \%)$ \\
\hline \multirow{4}{*}{ Sex } & Female & 177 & $(42.45 \%)$ & 140 & $(53.85 \%)$ & 100 & $(63.69 \%)$ \\
\hline & Male & 240 & $(57.55 \%)$ & 120 & $(46.15 \%)$ & 57 & $(36.31 \%)$ \\
\hline & Adenocarcinoma & 308 & $(73.86 \%)$ & 201 & $(77.31 \%)$ & 107 & $(68.15 \%)$ \\
\hline & Squamous & 33 & $(7.91 \%)$ & 14 & $(5.38 \%)$ & 19 & $(12.10 \%)$ \\
\hline \multirow{6}{*}{ Histology } & Adeno-squamous & 1 & $(0.24 \%)$ & 1 & $(0.38 \%)$ & & \\
\hline & $\begin{array}{l}\text { Large-cell } \\
\text { neuroendocrine }\end{array}$ & 2 & $(0.48 \%)$ & & & 2 & $(1.27 \%)$ \\
\hline & SCLC & 4 & $(0.96 \%)$ & 4 & $(1.54 \%)$ & & \\
\hline & NOS & 69 & $(16.55 \%)$ & 40 & $(15.38 \%)$ & 29 & $(18.47 \%)$ \\
\hline & Mutant & 62 & $(14.87 \%)$ & 47 & $(18.08 \%)$ & 15 & $(9.55 \%)$ \\
\hline & Wild type & 355 & $(85.13 \%)$ & 213 & $(81.92 \%)$ & 142 & $(90.45 \%)$ \\
\hline \multirow[t]{6}{*}{ EGFR Status } & $\begin{array}{l}\text { targetable EGFR } \\
\text { mutation }\end{array}$ & 41 & $(66.13 \%)$ & 33 & $(12.69 \%)$ & 8 & $(5.10 \%)$ \\
\hline & $\begin{array}{l}\text { targetable EGFR plus } \\
\text { resistance mutation } \\
\text { T790M }\end{array}$ & 3 & $(4.84 \%)$ & 3 & $(1.15 \%)$ & & \\
\hline & EGFR exon 20 insertion & 6 & $(9.68 \%)$ & 6 & $(2.31 \%)$ & & \\
\hline & $\begin{array}{l}\text { other / variant of } \\
\text { unknown significance }\end{array}$ & 12 & $(19.35 \%)$ & 5 & $(1.92 \%)$ & 7 & $(4.46 \%)$ \\
\hline & Mutant & 36 & $(8.63 \%)$ & 20 & $(7.69 \%)$ & 16 & $(10.19 \%)$ \\
\hline & Wild type & 381 & $(91.37 \%)$ & 240 & $(92.31 \%)$ & 141 & $(89.81 \%)$ \\
\hline \multirow{7}{*}{$B R A F$ Status } & V600E / class I* & 9 & $(25.00 \%)$ & 8 & $(3.08 \%)$ & 1 & $(0.64 \%)$ \\
\hline & non-V600E / class II * & 11 & $(30.56 \%)$ & 5 & $(1.92 \%)$ & 6 & $(3.82 \%)$ \\
\hline & $\begin{array}{l}\text { non-V600E / class III * } \\
\text { other mutation / variant }\end{array}$ & 5 & $(13.89 \%)$ & 3 & $(1.15 \%)$ & 2 & $(1.27 \%)$ \\
\hline & $\begin{array}{l}\text { of unknown } \\
\text { significance }\end{array}$ & 11 & $(30.56 \%)$ & 4 & $(1.54 \%)$ & 7 & $(4.46 \%)$ \\
\hline & Mutant & 41 & $(9.83 \%)$ & 32 & $(12.31 \%)$ & 9 & $(5.73 \%)$ \\
\hline & Wild type & 368 & $(88.25 \%)$ & 223 & $(85.77 \%)$ & 145 & $(92.36 \%)$ \\
\hline & n.d. & 8 & $(1.92 \%)$ & 5 & $(1.92 \%)$ & 3 & $(1.91 \%)$ \\
\hline \multirow[t]{6}{*}{ Gene Fusions } & $A L K$ translocation & 15 & $(36.59 \%)$ & 14 & $(5.38 \%)$ & 1 & $(0.64 \%)$ \\
\hline & ROS1 translocation & 2 & $(4.88 \%)$ & 1 & $(0.38 \%)$ & 1 & $(0.64 \%)$ \\
\hline & $\begin{array}{l}\text { RET translocation } \\
\text { other fusions / }\end{array}$ & 3 & $(7.32 \%)$ & 3 & $(1.15 \%)$ & & \\
\hline & $\begin{array}{l}\text { translocation of } \\
\text { unknown significance }\end{array}$ & 21 & $(51.22 \%)$ & 14 & $(5.38 \%)$ & 7 & $(4.46 \%)$ \\
\hline & $<1 \%$ & 123 & $(29.50 \%)$ & 79 & $(30.38 \%)$ & 44 & $(28.03 \%)$ \\
\hline & $\geq 1 \%$ and $<5 \%$ & 50 & $(11.99 \%)$ & 26 & $(10.00 \%)$ & 24 & $(15.29 \%)$ \\
\hline \multirow[t]{3}{*}{ PD-L1 TPS } & $\geq 5 \%$ and $<50 \%$ & 79 & $(18.94 \%)$ & 55 & $(21.15 \%)$ & 24 & $(15.29 \%)$ \\
\hline & $\geq 50 \%$ & 99 & $(23.74 \%)$ & 53 & $(20.38 \%)$ & 46 & $(29.30 \%)$ \\
\hline & n.d. & 66 & $(15.83 \%)$ & 47 & $(18.08 \%)$ & 19 & $(12.10 \%)$ \\
\hline
\end{tabular}

* BRAF mutations were classified based on Yao et al. [26].

\subsection{Turn-Around Time and HC NGS Workflow}

According to European guidelines, turn-around times for molecular testing in advanced lung cancer should take no longer than 10 working days $[27,28]$. We initially wanted to determine whether comprehensive testing including TMB estimation was feasible within this timeframe. To that end, we analyzed the first 115 cases for turn-around time and found that from $81.7 \%$ (94/115) of cases a result was sent to the treating physician within 10 working days (Figure 2). To further improve on the turn-around time, we looked at the steps responsible for possible delays more closely. The HC NGS 
workflow was subdivided into library preparation, sequencing, bioinformatics (data processing), and generation of a pathological report. Underlying causes for extended turn-around times in these 21 cases were delays in reporting (33\%) mainly due to complex genomic alterations; library preparation $(38 \%)$, such as insufficient DNA at intermediate steps of library preparation requiring some step to be repeated; other technical issues (19\%), such as operator or handling mistakes; or delays in data handling and bioinformatics due to server drop out (10\%) (Figure 2). Pre-analytics, including FFPE embedding, histological evaluation for tumor content and DNA extraction, required between 2 to 5 days in $80 \%$ of cases. More than 5 days were needed in $20 \%$ of samples due to delays in workflow (Figure 2). As only one HC NGS run was performed initially per week, the sample entry date also influenced overall TAT.

(A) Turn-around time TMB workflow in working days

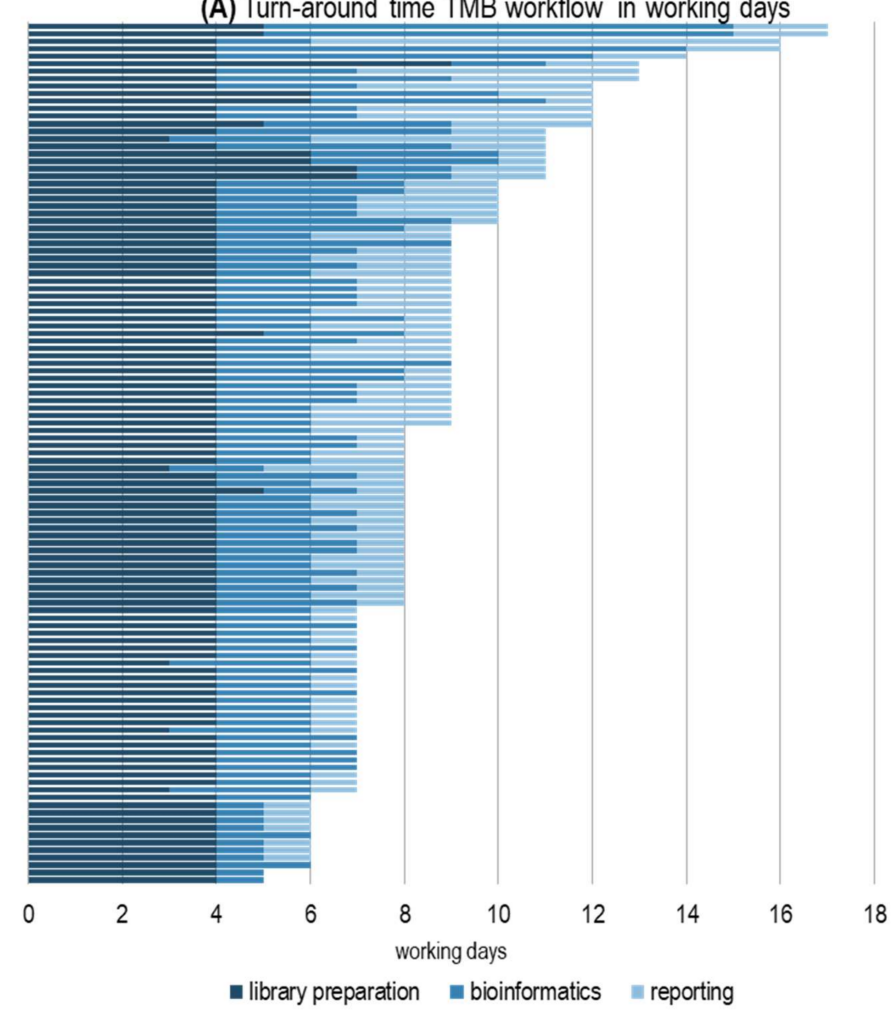

(B) Duration pre-analytics $(n=115)$

(C) Reasons for prolonged TAT $(n=21)$

(D) Dropout rate TMB workflow $(n=132)$
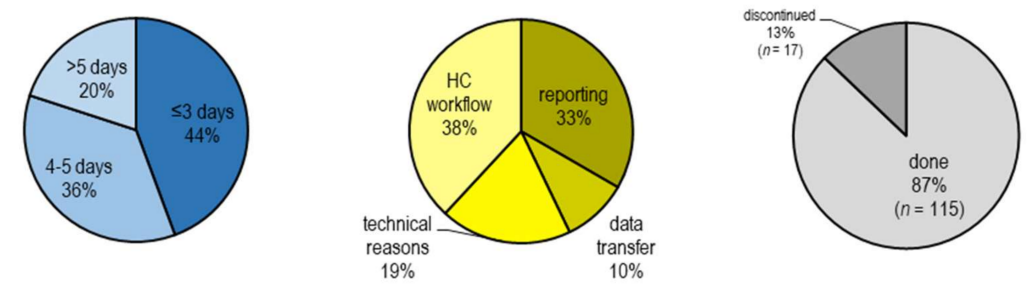

Figure 2. Turn-around time for TMB evaluation. (A) TAT in working days per case from start of HC NGS workflow to reporting; (B) Duration of pre-analytics including tissue embedding and DNA extraction; (C) Reasons for prolonged TAT ( $>10$ working days); (D) Percentage of cases not meeting quality criteria.

\subsection{Evaluation of Tumor Mutation Analysis in Routine Lab Samples}

\subsubsection{TMB in Relation to Age}

Based on these findings, we considered the assay set up sufficiently fast to continue using the assay for further routine diagnostics including targetable mutations. After a total of 417 were analyzed, 
we correlated patient-specific parameters with the observed TMB values. There appeared no significant age- $(p=0.476)$ or gender $(p=0.110)$-specific association with TMB (Figure 3$)$.

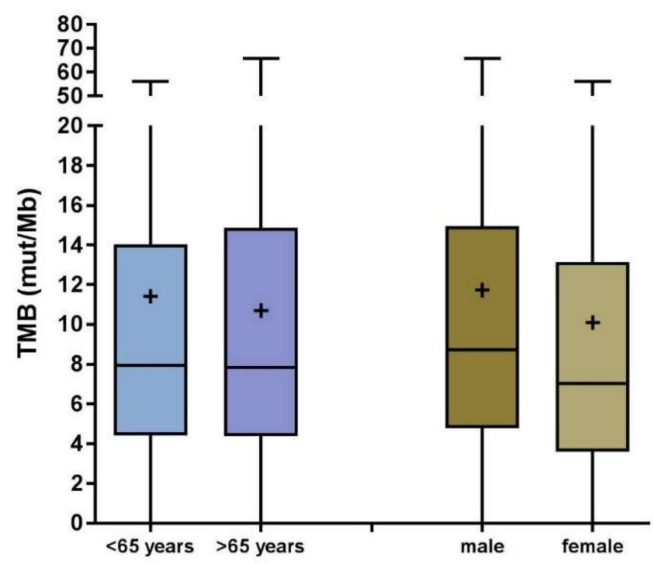

Figure 3. TMB status (mut/Mb) is not significantly correlated to age or gender. Boxplots are shown with the $95 \%$ confidence interval indicated by the box. Lines indicate the mean and + the median. Statistical analysis by Student $t$ test did not reveal significant differences in TMB between patients under or over 65 years of age $(p=0.476)$, nor between male and female patients $(p=0.110)$.

\subsubsection{TMB in Relation to Driver Mutations}

Recent reports suggest that the presence of typical driver alterations inversely correlates with the number of somatic tumor mutations $[29,30]$. In our cohort, targetable driver alterations (EGFR, ALK, ROS, BRAF V600E, RET) occurred at a combined frequency of $17.5 \%(73 / 417)$. The majority of this group of patients $62 / 73$ (84.9\%) showed a lower TMB, below a cut-off of $10 \mathrm{mut} / \mathrm{Mb}$, while only 11/73 $(15.1 \%)$ patients were in the TMB-high group $(\geq 10 \mathrm{mut} / \mathrm{Mb})$.

The most prevalent somatic mutations found were TP53 (237/417, 56.8\%), followed by KRAS (135/417, 32.4\%), KEAP1 (54/417, 15.8\%), STK11 (64/417, 15.3\%), EGFR (62/417, 14.9\%), ATM (46/417, $11.03 \%)$, and others. Gene amplifications were discovered in $45 / 417(10.8 \%)$ and gene fusions in $9.83 \%$ (41/417) of cases (Figure 4).

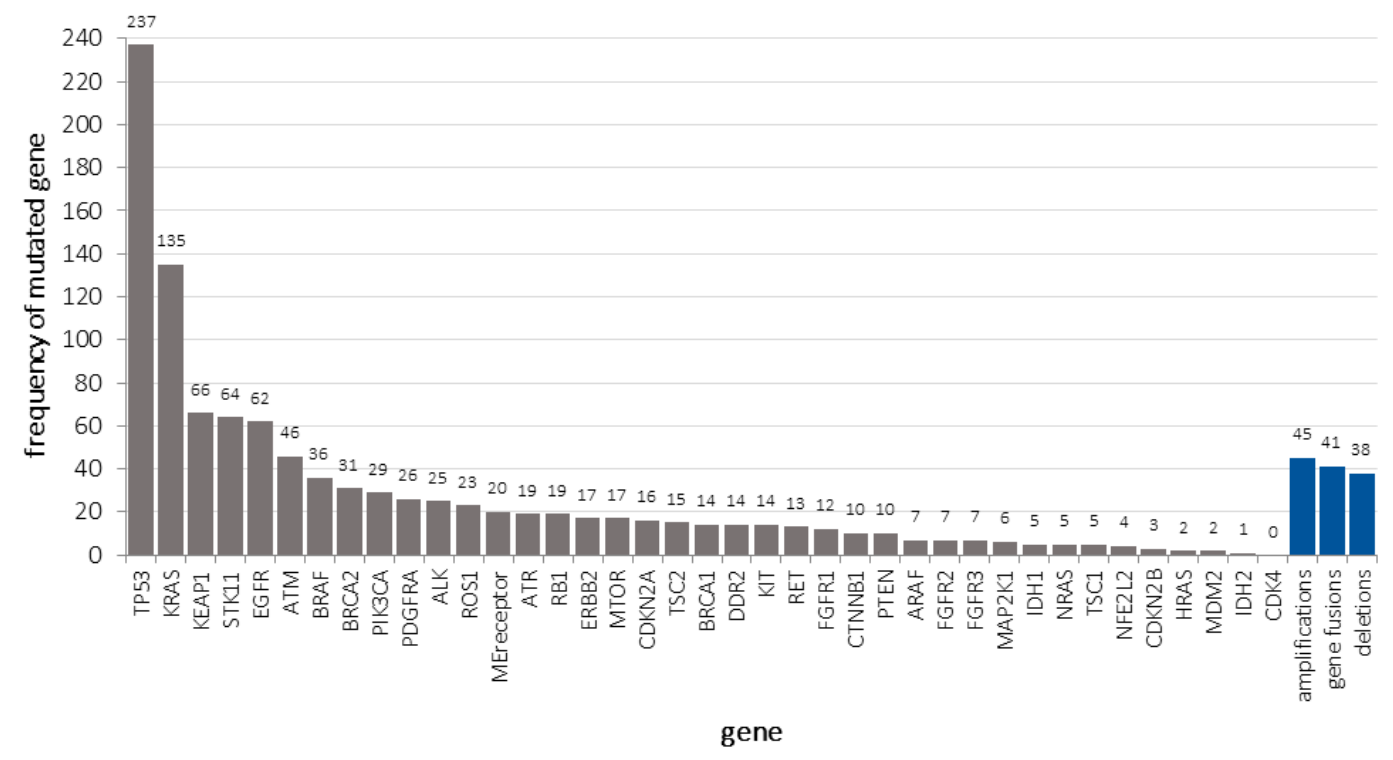

Figure 4. Number of tumors carrying non-synonymous gene mutations in descending order of frequency (grey), including gene amplifications, fusions, and deletions (blue). 


\subsubsection{TMB and PD-L1}

Data from the literature indicate that PD-L1 expression on tumor cells does not correlate with TMB and that TMB should therefore be considered as an independent predictive biomarker [31]. In line with this observation, we found no significant association between the TMB value and PD-L1 TPS (Figure 5). Further, we categorized the tumors on the basis of PD-L1 tumor proportion scores and observed no statistically significant association (as assessed by analysis of variance, ANOVA) of PD-L1 tumor expression and TPS scores $(p=0.798)$.

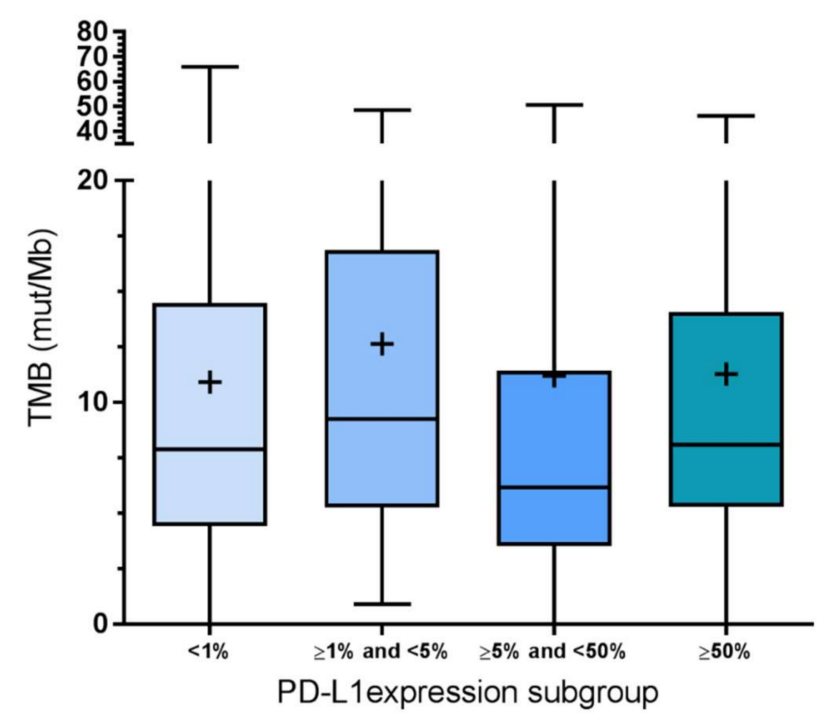

Figure 5. TMB is not different in the four groups of the PD-L1 tumor proportion score. Data are presented as box plots with a $95 \%$ confident interval. The line indicates the mean, the + indicates the median. Statistical analysis by ANOVA did not reveal significant $(p<0.05)$ differences in TMB between TPS groups.

\subsubsection{Correlation of Somatic Tumor Mutations and TMB}

It has been shown that the presence of targetable driving mutations, such as activating EGFR mutations, is associated only with limited responses to immune checkpoint inhibitors [32]. It has been postulated that this is due to low TMB. Particularly, EGFR and ALK mutations are more frequent in light or never smokers, further supporting this hypothesis [33]. Tumors characterized by a targetable driver alteration show significantly lower TMB compared to those lacking any driver alteration $(p<0.001)$. TMB in EGFR mutated tumors was significantly lower compared to TMB in KEAP1 $(p<0.01)$, ARID1A $(p<0.05)$, STK11 $(p<0.05)$, and POLE $(p<0.05)$ mutated tumors. Along those lines, TMB in ALK mutated tumors was significantly lower compared to KEAP1, ARID1A, POLE, and STK11 mutated tumors (each $p<0.05$ ) (see Figure 6). 


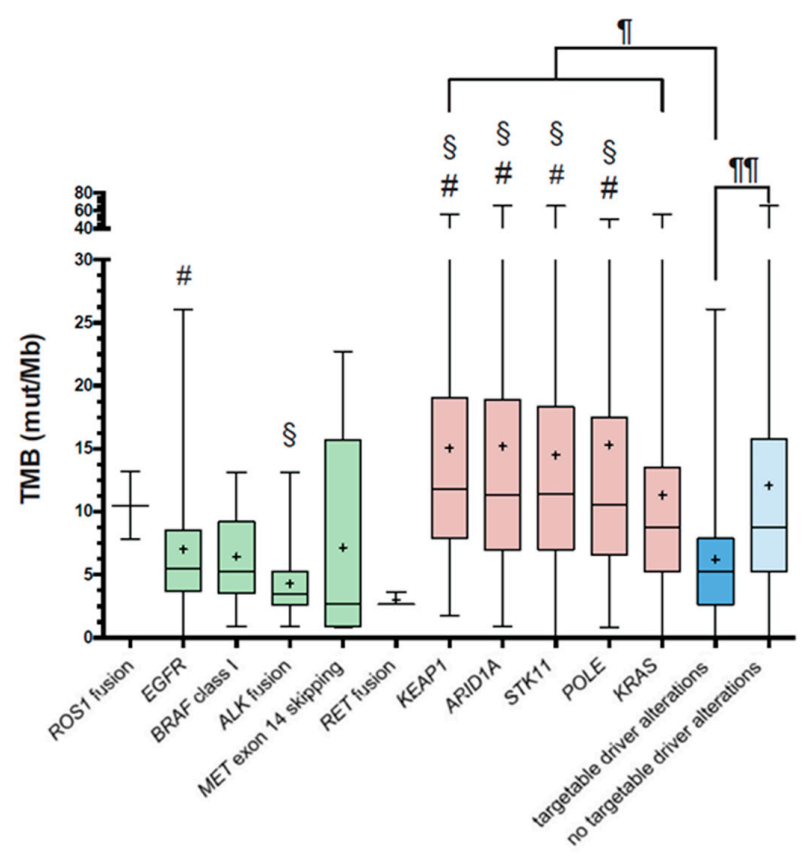

Figure 6. TMB and clinically relevant genetic alterations. For targetable driver alterations (green and blue), TKI-sensitive mutations were counted. Regarding KEAP1, ARID1A, STK11, and POLE, all non-synonymous aberrations (red) were considered. Data are presented as box plots with a $95 \%$ confident interval. The line indicates the mean, the + indicates the median. Statistical analysis by ANOVA revealed significant differences in TMB between EGFR and KEAP1, ARID1A, POLE, and STK11 (\#) mutated groups; between ALK fusion and KEAP1, ARID1A, POLE, and STK11 mutated groups (§); and between targetable driver mutations and all red groups (II); and lastly, between targetable and non-targetable driver mutation groups (III).

\subsection{Clinical Use of IO-Related Biomarkers in Clinical Decision-Making}

Recent publications suggest that STK11 and KEAP1 mutations might be associated with an inferior response to IO [34,35]. Contrarily, ARID1A mutations seem to confer positive prediction [36]. Based on these findings, we observe that clinicians are interested in additional biomarkers with predictive value for IO.

Inactivating mutations in STK11 were detected with a frequency of $11.3 \%$ (47/417) and for KEAP1 with 7.7\% (32/417). Functional mutations in ARID1A were found in 5.3\% (22/417) of patients. Interestingly, all these variants were associated with higher TMB values, indicating that particularly STK11 and KEAP1 are biomarkers independent from TMB.

\section{Discussion}

When TMB emerged as a potential predictive biomarker, it was considered to possibly be the most challenging and complex genomic biomarker to date. NGS-based sequencing of a minimum of one Megabase exonic territory in combination with advanced bioinformatics analyses is needed. The NEOplus v2 RUO ${ }^{\ddagger}$ panel has a total territory of $2.50 \mathrm{Mb}$, of which $1.14 \mathrm{Mb}$ are dedicated to TMB evaluation. The territory dedicated to TMB evaluation excludes typical cancer genes that are known to have a mutation bias. The need for a TMB territory size of greater than $1 \mathrm{Mb}$ is based on the data of Chalmers et al. and generally considered to be sufficient. This is supported by the comparisons of several TMB assays in the recently published German comparative TMB study [37]. There was considerable doubt whether speedy local TMB testing could be implemented and whether testing large number of patients was feasible. The initiatives of Quality in Pathology and Friends of Cancer Research jointly addressed the need for harmonization of TMB testing. In this study on a cohort of 417 lung cancer patients, we asked whether TMB can be assessed alongside other predictive genomic 
biomarkers needed for treatment stratification. We implemented an HC NGS assay into routine diagnostics, allowing for simultaneous detection of TMB and relevant aberrations including targetable driver mutations. TMB values correlated well with the F1Dx panel. These data are compatible with previously published data from a German harmonization trial [24,37].

The TAT proved to be in line with guidelines as $81.7 \%$ of cases met the required turn-around time of 10 working days. Comparing the NEOplus v2 RUO $¥$ HC assay to the F1Dx assay (used in CheckMate 227) revealed a high degree of association.

Samples analyzed in this study mainly consisted of adenocarcinomas; however, a significant proportion of biopsies were histologically classified in external pathologies and the histological subtype was not reported to us. Therefore, a relatively large proportion appeared as NOS; unfortunately, in most cases, tissue was not sufficient for reevaluation. TMB was not associated to age in our cohort, although previously, an age effect was published regarding other cancer entities [38]. TMB and PD-L1 expression was not associated, supporting the concept of two independent and therefore potentially additive biomarkers for immune oncological treatment. Male gender appears to be associated with higher TMB compared to females, potentially reflecting different smoking habits; however, this difference was not statistically significant. The frequency of targetable driver alterations (EGFR, ALK, ROS1, MET exon 14 skipping, RET, or BRAF V600E) was in line with the literature and their presence was significantly associated with a lower TMB value. In contrast, tumors that carried mutations in KRAS or genes assumed to cause primary resistance to IO (STK11, KEAP1, and POLE) showed higher TMB. In 2019, the complex CheckMate 227 trial using TMB as a co-primary endpoint in some subgroups could not convincingly show a role for TMB as a useful predictive biomarker. Since then retrospective analyses explored the use of pembrolizumab versus chemotherapy in all therapeutic lines, showing improvement in OS, PFS, and ORR for TMB-high patients [39,40].

Based on the KEYNOTE-158 trial, the FDA recently approved pembrolizumab monotherapy for patients with solid tumors and $\mathrm{TMB} \geq 10 \mathrm{mut} / \mathrm{Mb}$. The study included adults and pediatric patients with unresectable or metastatic disease with progression upon prior treatment or no alternative treatment options. This underlines the concept of TMB as a predictive biomarker [41].

\section{Materials and Methods}

\subsection{Selection of Patients}

Patients were retrospectively selected from our internal pathological documentation system based on the request for comprehensive molecular testing from 2018 to 2019. All samples represent tumor biopsies tested in a single institution (Institut für Hämatopathologie Hamburg, Germany). Use of anonymized patient data was reviewed by the local ethics committee (Ref number: WF-055/18 and WF-017/19.)

\subsection{PD-L1}

PD-L1 was stained immunohistochemically using the antibody clone 22C3 pharm Dx (Dako Omnis, 1:30 dilution) on the automated BenchMark Ultra platform (Roche Diagnostics) with positive controls of the spleen, tonsil, and placenta as part of a multi-tissue control. Scoring was conducted by board-certified and trained pathologists [42].

\subsection{Mutation Testing}

For molecular analysis, 3-10 5-10 $\mu \mathrm{m}$ micrometer formalin-fixed paraffin-embedded (FFPE) sections were prepared and tumor tissue was micro-dissected when the tumor content was below 10\%. DNA was extracted semi-automated (Maxwellß16, Promega), and 400ng of input DNA was sonographically sheared (Covarisß) into approximately 200-bp double-stranded fragments. Hereafter, adapters were ligated, and genomic regions of interest were enriched using complementary bait sequences. During this hybrid capture, the selected baits ensure optimal coverage of all relevant 
genomic regions, including 340 genes in a $1.14 \mathrm{Mb}$ complete genomic territory size. Following the enrichment, the targeted fragments were clonally amplified and sequenced with next-generation sequencing (NextSeq 500/550, Illumina). Point mutations, small insertions and deletions, copy number alterations, and rearrangement/gene fusions were identified with NEO New Oncology's proprietary computational biology analysis pipeline and analyzed using the NEO diagnosis software.

\section{4. $T M B$}

For TMB, the number of somatic mutations detected within $1.14 \mathrm{Mb}$ of the genome were quantified and that value extrapolated to the whole exome using a validated algorithm (NEO New Oncology). Alterations known to be included in genomic databases, such as Single Nucleotide Polymorphism Database (dbSNP) or Exome Aggregation Consortium (ExAc), were excluded.

TMB was calculated from genomic alterations identified by the bioinformatics pipeline. Single nucleotide changes with predicted missense, nonsense, silent, nonstop consequences, and small insertions/deletions with in-frame or frameshift insertion/deletion consequences were considered for TMB calculation. Variants with an allelic frequency of at least $5 \%$ (for LOD 0.05 value) or $10 \%$ (for LOD 0.1 value) were included and frequent germ line variants present in ExAc and dbSNP were not considered. The TMB value was provided as mutations per Megabase (mut/Mb).

\subsection{Statistical Analysis}

Descriptive statistics were used. Differences between groups were tested with parametric or non-parametric methods depending on the distribution. Box plots were generated using GraphPad Prism 7.04 (GraphPad Software, Inc., San Diego, CA) and statistical significance assessed by Student $t$ and ANOVA (Alpha level 0.05). For Figure 1, Pearson correlation was performed using Microsoft Excel.

\section{Conclusions}

We showed that routine diagnostics of highly complex biomarkers, such as TMB, is feasible today and that a central industrial testing facility is not required. This is a highly important finding given that clinical trial results using TMB as a predictive biomarker are promising and FDA approval is currently pending. However, at this point in time, data are still not fully mature and further validation is required.

Author Contributions: Conceptualization, L.D., F.G., M.T. and L.C.H.; Data curation, M.F., B.J., S.S. (Stefanie Schmidt), R.M., H.J.M.G. and F.G.; Formal analysis, B.J. and R.M.; Investigation, M.T.; Methodology, S.S. (Stefanie Schatz), S.S. (Stefanie Schmidt) and E.-M.W.; Resources, M.K., C.W., F.G., P.H. and M.T.; Software, E.-M.W.; Supervision, F.G., M.T. and L.C.H.; Visualization, S.S. (Stefanie Schatz), M.F. and B.J.; Writing—original draft, S.S. (Stefanie Schatz), M.F. and B.J.; Writing—review \& editing, H.O.R., H.J.M.G., L.D., M.K., C.W., F.G., P.H. and L.C.H.. All authors have read and agreed to the published version of the manuscript.

Funding: This research received no external funding.

Acknowledgments: We thank Birgit Brussé for data documentation and Sabine Bleicher for extraordinary technical support.

Conflicts of Interest: P.H. Honorarium: Boehringer Ingelheim, Pfizer, Takeda, Roche Pharma, Lilly, BMS, MSD, AstraZeneca, Abbvie; F.G. Honorarium: Astra Zeneca, Boehringer Ingelheim, BMS, Celgene, Lilly, MSD, Novartis, Pfizer, Roche, Takeda, Ariad, Abbvie, Siemens; M.T.: Honorarium: AstraZeneca, Boehringer Ingelheim, Novartis, Roche, Pfizer, Takeda, BMS., MSD.; M.F.: Honorarium: Boehringer Ingelheim, AstraZeneca, BMS, Novartis, Roche; L.C.H.: Honorarium: Boehringer Ingelheim, Pfizer, BMS., MSD., AstraZeneca. All other authors do not declare any conflict of interest.

\section{Appendix A}

List of genes included in NEOplus v2 RUO ${ }^{\ddagger}$. NEOplus v2 RUO $\ddagger$ detects point mutations in 330 genes and small insertions and deletions in 325 genes, as well as copy number changes in 230 genes. In addition, gene fusions are detected in 16 genes.

$\ddagger$ For Research Use Only. Not for use in diagnostic procedures. 
Point Mutations, small Insertions and Deletions

ABL1, ABL2, ACVR1B, AKT1, AKT2, AKT3, ALK, ALMS1, AMER1, APC, APLNR, AR, ARAF, ARHGEF12, ARID1A, ARID1B, ARID2, ASXL1, ATAD5*, ATM, ATR, ATRX, AURKA, AURKB, AXIN2, AXL, B2M, BAP1, BARD1, BCL2, BCL2L1, BCL6, BLM, BMPR1A, BMS1, BRAF, BRCA1, BRCA2, BRD2, BRD3, BRD4, BRIP1, BTK, BUB1B, CARD11, CASP8, CBFB, CBL, CCND1, CCND2, CCNE1, CD274, CD58, CD79A, CD79B, CDC73, CDH1, CDK12, CDK4, CDK6, CDK8, CDKN1A, CDKN1B, CDKN2A, CDKN2B, CDKN2C, CEBPA, CHD4, CHEK1, CHEK2, CIC, CLSPN, CREBBP, CRKL, CSF1R, CTCF, CTNNA1, CTNNB1, CUL3, DAXX, DCUN1D4, DDB2, DDR2, DICER1, DOT1L, EGFR, EMSY, EP300, EPHA3, EPHA5, EPHA7, EPHB1, ERBB2, ERBB3, ERBB4, ERCC3, ERCC4, ERCC5, ERCC6, ERRFI1, ESR1, EZH2, FANCA, FANCB, FANCC, FANCD2, FANCE, FANCF, FANCG, FANCI, FANCL, FANCM, FAS, FAT1, FBXW7, FGFR1, FGFR2, FGFR3, FGFR4, FLCN, FLT1, FLT3, FOXO3, FOXP1, FRS2, GATA1, GEN1, GLI1, GNA11, GNA13, GNAI2, GNAQ, GNAS, GNAT2, GSK3B, H3F3A, H3F3B, HDAC2, HGF, HLTF, HRAS, HSP90AA1, IDH1, IDH2, IFNGR1, IFNGR2, IGF1R, IGF2, IGF2R, IKBKB, IKBKE, IL21R, INHBA, INPP4B, IRF1, IRF2, IRF4, JAK1, JAK2, JAK3, JUN, KAT6A*, KDR, KEAP1, KIT, KMT2A, KMT2B, KMT2C, KMT2D, KRAS, KSR1, LRP1B, LYN, MAD2L1, MAGI2, MAP2K1, MAP2K3, MAP2K4, MAP3K1, MCL1, MDM2, MDM4, MED12, MEN1, MET, MITF, MLANA, MLH1, MLH3, MPL, MRE11, MSH2, MSH3, MSH5, MSH6, MST1R, MTOR, MUTYH, MYC, MYCL, MYCN, NBN, NCOA3, NCOA4, NF1, NF2, NFE2L2, NFKBIA, NOTCH2, NOTCH4, NPM1, NRAS, NSD1, NTRK1, NTRK2, NTRK3, PALB2, PBRM1, PDCD1LG2, PDGFRA, PDGFRB, PDK1, PIK3C2B, PIK3CA, PIK3CB, PIK3CD, PIK3CG, PIK3R1, PIK3R2, PLCG2, PMS2, POLD1, POLE, POLG, POLH, POT1, PPM1D, PPP2R1A, PRDM1, PREX2, PRKCI, PRKDC, PRKN, PSMB5, PTCH1, PTEN, PTPN11, PTPRC, PTPRK, PTPRT, QKI, RAC1, RAD50, RAD51, RAD51B, RAD51C, RAD51D, RAD54L, RAD54L2, RAF1, RANBP2, RARA, RB1, RECQL4, RET, REV3L, RICTOR, RIT1, RNF43, RNPS1, ROS1, RPL10A, RPL23, RPTOR, SDHA*, SDHAF2, SDHB, SDHC, SDHD, SERPINB3, SERPINB4, SETD1A*, SETD1B, SETD2, SF3B1, SLIT2, SLX4, SMAD2, SMAD3, SMAD4, SMARCA4, SMARCB1, SMO, SOX2, SPEN, SPOP, SRP54, STAG2, STAT3, STK11, SUFU, SYK, TAF3, TAP1, TAP2, TAPBP, TCF7L2, TGFBR2, TNFAIP3, TNFRSF14, TOP1, TOP2A, TP53, TP53BP1, TRRAP, TSC1, TSC2, TSHR, U2AF1, U2AF1L5, VEGFA, VHL, WISP3, WRN, WT1, XPA, XPC, XRCC1, ZFHX3*, ZNF217

* Detection of small insertions and deletions in ATAD5, KAT6A, SDHA, SETD1A, ZFHX3 not possible.

Copy Number Changes

ABL1, AKT1, AKT2, AKT3, ALK, ALMS1, AMER1, APC, APLNR, AR, ARAF, ARID1A, ARID1B, ARID2, ASXL1, ATAD5, ATM, ATR, ATRX, AURKA, AXIN2, AXL, BARD1, BCL6, BLM, BMPR1A, BMS1, BRAF, BRCA1, BRCA2, BRD2, BRD3, BRD4, BRIP1, BTK, BUB1B, CARD11, CASP8, CCND1, CCNE1, CD274, CDC73, CDH1, CDK12, CDK4, CDK6, CDK8, CDKN2A, CDKN2B, CHD4, CIC, CLSPN, CREBBP, CSF1R, CTNNA1, CTNNB1, CUL3, DAXX, DDB2, DDR2, DICER1, DOT1L, EGFR, EMSY, EP300, EPHA3, EPHB1, ERBB2, ERBB3, ERBB4, ERCC3, ERCC4, ERCC5, ERCC6, ERRFI1, ESR1, EZH2, FANCA, FANCB, FANCC, FANCD2, FANCE, FANCI, FANCL, FANCM, FAS, FAT1, FGF19\$, FGF23\#, FGF3\$, FGF4\$, FGF6\#, FGFR1, FGFR2, FGFR3, FGFR4, FLT1, FLT3, FOXO3, FOXP1, FRS2, GATA1, GLI1, GSK3B, HDAC2, IDH1, IDH2, IFNGR1, IKBKB, IKBKE, IL21R, INHBA, INPP4B, IRF1, IRF2, IRF4, JAK1, JAK2, JAK3, KAT6A, KDR, KEAP1, KIT, KRAS, LRP1B, LYN, MAGI2, MAP2K1, MDM2, MDM4, MED12, MEN1, MET, MITF, MLH1, MPL, MRE11, MSH2, MSH3, MSH5, MSH6, MST1R, MUTYH, MYC, MYCL, MYCN, NBN, NCOA3, NCOA4, NF1, NF2, NFE2L2, NPM1, NSD1, NTRK1, NTRK2, NTRK3, PALB2, PBRM1, PDGFRA, PDGFRB, PDK1, PIK3C2B, PIK3CA, PIK3CB, PIK3CD, PIK3CG, PIK3R1, PIK3R2, PMS2, POLD1, POLE, POLG, POLH, PPM1D, PRDM1, PTCH1, PTEN, PTPN11, PTPRK, QKI, RAD50, RAD51, RAD51B, RAD51C, RAD51D, RAD54L, RAD54L2, RAF1, RANBP2, RARA, RB1, REV3L, RICTOR, RIT1, RNF43, ROS1, RPTOR, SETD1A, SETD1B, SETD2, SF3B1, SLIT2, SLX4, SMAD2, SMAD3, SMAD4, SMARCA4, SMO, SPOP, STAG2, STAT3, STK11, SYK, TAP1, TAP2, TAPBP, TGFBR2, TNFAIP3, TOP1, TOP2A, TP53, TP53BP1, TRRAP, TSC1, TSC2, VEGFA, WRN, XPC, XRCC1 
$\$$ genes considered as one cluster \# genes considered as one cluster Gene Fusions

ALK, BRAF, CD74, EML4, ETV6 (NTRK3 Fusion), FGFR1, FGFR2, FGFR3, KIF5B, MET, NRG1, NTRK1, PDGFRA, RET, ROS1

\section{References}

1. Kerr, K.M. The PD-L1 Immunohistochemistry biomarker: Two steps forward, one step back? J. Thorac. Oncol. 2018, 13, 291-294. [CrossRef] [PubMed]

2. Hirsch, F.R.; McElhinny, A.; Stanforth, D.; Ranger-Moore, J.; Jansson, M.; Kulangara, K.; Richardson, W.; Towne, P.; Hanks, D.; Vennapusa, B.; et al. PD-L1 Immunohistochemistry assays for lung cancer: Results from phase 1 of the blueprint PD-L1 IHC assay comparison project. J. Thorac. Oncol. 2017, 12, 208-222. [CrossRef] [PubMed]

3. Rimm, D.; Han, G.; Taube, J.M.; Yi, E.S.; Bridge, J.A.; Flieder, U.B.; Homer, R.; West, W.W.; $\mathrm{Wu}, \mathrm{H} . ;$ Roden, A.C.; et al. A Prospective, Multi-institutional, pathologist-based assessment of 4 immunohistochemistry assays for PD-L1 expression in non-small cell lung cancer. JAMA Oncol. 2017, 3, 1051-1058. [CrossRef] [PubMed]

4. Ratcliffe, M.J.; Sharpe, A.; Midha, A.; Barker, C.; Scott, M.; Scorer, P.; Al-Masri, H.; Rebelatto, M.C.; Walker, J. Agreement between programmed cell death ligand-1 diagnostic assays across multiple protein expression cutoffs in non-small cell lung cancer. Clin. Cancer Res. 2017, 23, 3585-3591. [CrossRef]

5. Tsao, M.-S.; Kerr, K.M.; Kockx, M.; Beasley, M.-B.; Borczuk, A.C.; Botling, J.; Bubendorf, L.; Chirieac, L.; Chen, G.; Chou, T.-Y.; et al. PD-L1 Immunohistochemistry comparability study in real-life clinical samples: Results of blueprint phase 2 project. J. Thorac. Oncol. 2018, 13, 1302-1311. [CrossRef]

6. Hellmann, M.D.; Rizvi, N.A.; Goldman, J.W.; Gettinger, S.N.; Borghaei, H.; Brahmer, J.A.; Ready, N.E.; Gerber, D.E.; Chow, L.Q.; Juergens, R.A.; et al. Nivolumab plus ipilimumab as first-line treatment for advanced non-small-cell lung cancer (CheckMate 012): Results of an open-label, phase 1, multicohort study. Lancet Oncol. 2017, 18, 31-41. [CrossRef]

7. Cristescu, R.; Mogg, R.; Ayers, M.; Albright, A.; Murphy, E.; Yearley, J.; Sher, X.; Liu, X.Q.; Lu, H.; Nebozhyn, M.; et al. Pan-tumor genomic biomarkers for PD-1 checkpoint blockade-based immunotherapy. Science 2018, 362, eaar3593. [CrossRef]

8. Chalmers, Z.R.; Connelly, C.F.; Fabrizio, D.; Gay, L.; Ali, S.M.; Ennis, R.; Schrock, A.; Campbell, B.; Shlien, A.; Chmielecki, J.; et al. Analysis of 100,000 human cancer genomes reveals the landscape of tumor mutational burden. Genome Med. 2017, 9, 34. [CrossRef]

9. Schumacher, T.N.; Schreiber, R.D. Neoantigens in cancer immunotherapy. Science 2015, 348, 69-74. [CrossRef]

10. Gubin, M.M.; Artyomov, M.N.; Mardis, E.R.; Schreiber, R.D. Tumor neoantigens: Building a framework for personalized cancer immunotherapy. J. Clin. Investig. 2015, 125, 3413-3421. [CrossRef]

11. Grizzi, G.; Caccese, M.; Gkountakos, A.; Carbognin, L.; Tortora, G.; Bria, E.; Pilotto, S. Putative predictors of efficacy for immune checkpoint inhibitors in non-small-cell lung cancer: Facing the complexity of the immune system. Expert Rev. Mol. Diagn. 2017, 17, 1055-1069. [CrossRef] [PubMed]

12. Chabanon, R.M.; Pedrero, M.; Lefebvre, C.; Marabelle, A.; Soria, J.-C.; Postel-Vinay, S. Mutational landscape and sensitivity to immune checkpoint blockers. Clin. Cancer Res. 2016, 22, 4309-4321. [CrossRef] [PubMed]

13. Luksza, M.; Riaz, N.; Makarov, V.; Balachandran, V.P.; Hellmann, M.D.; Solovyov, A.; Rizvi, N.A.; Merghoub, T.; Levine, A.J.; Chan, T.A.; et al. A neoantigen fitness model predicts tumour response to checkpoint blockade immunotherapy. Nature 2017, 551, 517-520. [CrossRef] [PubMed]

14. Rizvi, H.; Sanchez-Vega, F.; La, K.; Chatila, W.; Jonsson, P.; Halpenny, D.; Plodkowski, A.; Long, N.; Sauter, J.L.; Rekhtman, N.; et al. Molecular determinants of response to anti-programmed cell death (pd)-1 and anti-programmed death-ligand 1 (PD-L1) blockade in patients with non-small-cell lung cancer profiled with targeted next-generation sequencing. J. Clin. Oncol. 2018, 36, 633-641. [CrossRef] [PubMed]

15. Carbone, D.P.; Reck, M.; Paz-Ares, L.; Creelan, B.; Horn, L.; Steins, M.; Felip, E.; Heuvel, M.M.V.D.; Ciuleanu, T.-E.; Badin, F.; et al. First-line nivolumab in stage IV or recurrent non-small-cell lung cancer. N. Engl. J. Med. 2017, 376, 2415-2426. [CrossRef] [PubMed] 
16. Hellmann, M.D.; Nathanson, T.; Rizvi, H.; Creelan, B.C.; Sanchez-Vega, F.; Ahuja, A.; Ni, A.; Novik, J.B.; Mangarin, L.M.; Abu-Akeel, M.; et al. Genomic features of response to combination immunotherapy in patients with advanced non-small-cell lung cancer. Cancer Cell 2018, 33, 843-852.e4. [CrossRef]

17. Boumber, Y. Tumor mutational burden (TMB) as a biomarker of response to immunotherapy in small cell lung cancer. J. Thorac. Dis. 2018, 10, 4689-4693. [CrossRef]

18. Hellmann, M.D.; Ciuleanu, T.-E.; Pluzanski, A.; Lee, J.S.; Otterson, G.A.; Audigier-Valette, C.; Minenza, E.; Linardou, H.; Burgers, S.; Salman, P.; et al. Nivolumab plus ipilimumab in lung cancer with a high tumor mutational burden. N. Engl. J. Med. 2018, 378, 2093-2104. [CrossRef]

19. Samstein, R.M.; Lee, C.-H.; Shoushtari, A.N.; Hellmann, M.D.; Shen, R.; Janjigian, Y.Y.; Barron, D.A.; Zehir, A.; Jordan, E.; Omuro, A.; et al. Tumor mutational load predicts survival after immunotherapy across multiple cancer types. Nat. Genet. 2019, 51, 202-206. [CrossRef]

20. Gandhi, L.; Rodríguez-Abreu, D.; Gadgeel, S.; Esteban, E.; Felip, E.; De Angelis, F.; Dómine, M.; Clingan, P.; Hochmair, M.J.; Powell, S.F.; et al. Pembrolizumab plus chemotherapy in metastatic non-small-cell lung cancer. N. Engl. J. Med. 2018, 378, 2078-2092. [CrossRef]

21. Borghaei, H.; Langer, C.J.; Gadgeel, S.; Papadimitrakopoulou, V.A.; Patnaik, A.; Powell, S.F.; Gentzler, R.D.; Martins, R.G.; Stevenson, J.P.; Jalal, S.I.; et al. 24-month overall survival from KEYNOTE-021 cohort g: Pemetrexed and carboplatin with or without pembrolizumab as first-line therapy for advanced nonsquamous non-small cell lung cancer. J. Thorac. Oncol. 2019, 14, 124-129. [CrossRef] [PubMed]

22. Kowanetz, M.; Zou, W.; McCleland, M.; Gandara, D.; Gadgeel, S.; Rittmeyer, A.; Barlési, F.; Park, K.; Shames, D.; Koeppen, H.; et al. MA 05.09 Pre-existing immunity measured by teff gene expression in tumor tissue is associated with atezolizumad efficacy in NSCLC. J. Thorac. Oncol. 2017, 12, S1817-S1818. [CrossRef]

23. Nahar, R.; Zhai, W.; Zhang, T.; Takano, A.; Khng, A.J.; Lee, Y.Y.; Liu, X.; Lim, C.H.; Koh, T.P.T.; Aung, Z.W.; et al. Elucidating the genomic architecture of Asian EGFR-mutant lung adenocarcinoma through multi-region exome sequencing. Nat. Commun. 2018, 9, 216. [CrossRef] [PubMed]

24. Stenzinger, A.; Allen, J.; Maas, J.; Stewart, M.D.; Merino, D.M.; Wempe, M.M.; Dietel, M. Tumor mutational burden standardization initiatives: Recommendations for consistent tumor mutational burden assessment in clinical samples to guide immunotherapy treatment decisions. Genes Chromosom. Cancer 2019, 58, 578-588. [CrossRef]

25. Addeo, A.; Banna, G.L.; Weiss, G.J. Tumor mutation burden-from hopes to doubts. JAMA Oncol. 2019, 5, 934-935. [CrossRef]

26. Yao, Z.; Yaeger, R.; Rodrik-Outmezguine, V.S.; Tao, A.; Torres, N.M.; Chang, M.T.; Drosten, M.; Zhao, H.; Cecchi, F.; Hembrough, T.; et al. Tumours with class 3 BRAF mutants are sensitive to the inhibition of activated RAS. Nature 2017, 548, 234-238. [CrossRef]

27. Metastatic Non-Small-Cell Lung Cancer: ESMO Clinical Practice Guidelines for Diagnosis, Treatment and Follow-up. Clinical Practice Guidelines [Guidelines]. 2018. Available online: https://www. esmo.org/Guidelines/Lung-and-Chest-Tumours/Metastatic-Non-Small-Cell-Lung-Cancer (accessed on 26 January 2019).

28. Prävention, Diagnostik, Therapie und Nachsorge des Lungenkarzinoms, Langversion 1.0. Leitlinienprogramm Onkologie. 2018. Available online: http://leitlinienprogramm-onkologie.de/ Lungenkarzinom.98.0.html (accessed on 1 February 2018).

29. Offin, M.; Rizvi, H.; Tenet, M.; Ni, A.; Sanchez-Vega, F.; Li, B.T.; Drilon, A.; Kris, M.G.; Rudin, C.M.; Schultz, N.; et al. Tumor mutation burden and efficacy of EGFR-tyrosine kinase inhibitors in patients with EGFR-mutant lung cancers. Clin. Cancer Res. 2019, 25, 1063-1069. [CrossRef]

30. Spigel, D.R.; Schrock, A.B.; Fabrizio, D.; Frampton, G.M.; Sun, J.; He, J.; Gowen, K.; Johnson, M.L.; Bauer, T.M.; Kalemkerian, G.P.; et al. Total mutation burden (TMB) in lung cancer (LC) and relationship with response to PD-1/PD-L1 targeted therapies. J. Clin. Oncol. 2016, 34, 9017. [CrossRef]

31. Yarchoan, M.; Albacker, L.A.; Hopkins, A.C.; Montesion, M.; Murugesan, K.; Vithayathil, T.T.; Zaidi, N.; Azad, N.S.; Laheru, D.A.; Frampton, G.M.; et al. PD-L1 expression and tumor mutational burden are independent biomarkers in most cancers. JCI Insight 2019, 4. [CrossRef] 
32. Reck, M.; Schenker, M.; Lee, K.H.; Provencio, M.; Nishio, M.; Lesniewski-Kmak, K.; Sangha, R.; Ahmed, S.; Raimbourg, J.; Feeney, K.; et al. Nivolumab plus ipilimumab versus chemotherapy as first-line treatment in advanced non-small-cell lung cancer with high tumour mutational burden: Patient-reported outcomes results from the randomised, open-label, phase III CheckMate 227 trial. Eur. J. Cancer 2019, 116, 137-147. [CrossRef]

33. Chapman, A.M.; Sun, K.Y.; Ruestow, P.; Cowan, D.M.; Madl, A.K. Lung cancer mutation profile of EGFR, ALK, and KRAS: Meta-analysis and comparison of never and ever smokers. Lung Cancer 2016, 102, 122-134. [CrossRef] [PubMed]

34. Skoulidis, F.; Goldberg, M.E.; Greenawalt, D.M.; Hellmann, M.D.; Awad, M.M.; Gainor, J.F.; Schrock, A.B.; Hartmaier, R.J.; Trabucco, S.E.; Gay, L.; et al. STK11/LKB1 Mutations and PD-1 Inhibitor Resistance in KRAS-Mutant Lung Adenocarcinoma. Cancer Discov. 2018, 8, 822-835. [CrossRef] [PubMed]

35. Lagos, G.G.; Izar, B.; Rizvi, N.A. Beyond Tumor PD-L1: Emerging genomic biomarkers for checkpoint inhibitor immunotherapy. Am. Soc. Clin. Oncol. Educ. Book 2020, 40, e47-e57. [CrossRef] [PubMed]

36. Rizvi, N.; Cho, B.; Reinmuth, N.; Lee, K.; Luft, A.; Ahn, M.; Papadimitrakopoulou, V.; Heymach, J.; Scheuring, U.; Higgs, B.; et al. OA04.07 Mutations associated with sensitivity or resistance to immunotherapy in mNSCLC: Analysis from the MYSTIC trial. J. Thorac. Oncol. 2019, 14, S217. [CrossRef]

37. Stenzinger, A.; Endris, V.; Budczies, J.; Merkelbach-Bruse, S.; Kazdal, D.; Dietmaier, W.; Pfarr, N.; Siebolts, U.; Hummel, M.; Herold, S.; et al. Harmonization and standardization of panel-based tumor mutational burden measurement: Real-world results and recommendations of the quality in pathology study. J. Thorac. Oncol. 2020. [CrossRef] [PubMed]

38. Klebanov, N.; Artomov, M.; Goggins, W.B.; Daly, E.; Daly, M.J.; Tsao, H. Burden of unique and low prevalence somatic mutations correlates with cancer survival. Sci. Rep. 2019, 9, 4848. [CrossRef]

39. Herbst, R.S.; Baas, P.; Kim, N.-W.; Felip, E.; Perez-Gracia, J.L.; Han, J.-Y.; Molina, J.; Kim, J.-H.; Arvis, C.D.; Ahn, M.-J.; et al. Pembrolizumab versus docetaxel for previously treated, PD-L1-positive, advanced non-small-cell lung cancer (KEYNOTE-010): A randomised controlled trial. Lancet 2016, 387, 1540-1550. [CrossRef]

40. Mok, T.S.; Wu, Y.-L.; Kudaba, I.; Kowalski, D.M.; Cho, B.C.; Turna, H.Z.; Castro, G.; Srimuninnimit, V.; Laktionov, K.P.; Bondarenko, I.; et al. Pembrolizumab versus chemotherapy for previously untreated, PD-L1-expressing, locally advanced or metastatic non-small-cell lung cancer (KEYNOTE-042): A randomised, open-label, controlled, phase 3 trial. Lancet 2019, 393, 1819-1830. [CrossRef]

41. Piha-Paul, S.A.; Oh, D.-Y.; Ueno, M.; Malka, D.; Chung, H.C.; Nagrial, A.; Kelley, R.K.; Ros, W.; Italiano, A.; Nakagawa, K.; et al. Efficacy and safety of pembrolizumab for the treatment of advanced biliary cancer: Results from the KEYNOTE-158 and KEYNOTE-028 studies. Int. J. Cancer 2020. [CrossRef]

42. Scheel, A.H.; Dietel, M.; Heukamp, L.C.; Jöhrens, K.; Kirchner, T.; Reu, S.; Rüschoff, J.; Schildhaus, H.U.; Schirmacher, P.; Tiemann, M.; et al. Predictive PD-L1 immunohistochemistry for non-small cell lung cancer: Current state of the art and experiences of the first German harmonization study. Pathologe 2016, 37, 557-567. [CrossRef]

(C) 2020 by the authors. Licensee MDPI, Basel, Switzerland. This article is an open access article distributed under the terms and conditions of the Creative Commons Attribution (CC BY) license (http://creativecommons.org/licenses/by/4.0/). 